\title{
Joint Congestion Control and Routing Subject to Dynamic Interruptions in Cognitive Radio Networks
}

\author{
Husheng Li \\ Department of EECS \\ University of Tennessee \\ Knoxville, TN 37996 \\ Email: husheng@eecs.utk.edu
}

\author{
Lijun Qian \\ Department of ECE \\ Prairie View A\&M University, Texas A\&M University System \\ Prairie View, TX 77446 \\ Email: liqian@pvamu.edu
}

\begin{abstract}
Cognitive radio networks suffer from dynamic interruptions from primary users. The joint congestion control and routing are tackled using stochastic control techniques. Centralized dynamic programming is applied for the primal optimization, which provides a performance upper bound. $Q$ learning is applied when the primary user knowledge is unknown. Dual optimization based decomposition is used to decentralize the stochastic control. A heuristic scheme based on the limited lookahead policy (LLP) and binary pricing is proposed to tackle the prohibitive difficulty in the dual optimization. Numerical simulation shows that the proposed algorithms achieve the optimal or near-optimal performance.
\end{abstract}

\section{INTRODUCTION}

Cognitive radio networks, illustrated in Fig. 1, are attracting more and more studies in recent years due to its capability of alleviating the problem of spectrum underutilization. The fundamental change of spectrum access incurs significant challenges to all layers in cognitive radio networks. Researchers have proposed new algorithms and protocols to combat the new challenges, e.g. Quality of Service (QoS) aware scheduling [11] [12], spectrum-aware routing [7] [9] [14], distributed resource allocation [10] and a new TCP protocol incorporating the activities of primary users [3].

As the new scheme of spectrum access incurs significant impacts on all layers, it yields a better performance to design the network across different layers of cognitive radio networks. Note that the cross-layer design has been considered for scheduling [11] and routing [9] in cognitive radio networks. However, no uniform mathematical framework is proposed in these studies. Note that the cross-layer design framework has been widely used for analyzing and designing networks in the first decade of this century [2] [5] [6]. For example, the joint congestion control and routing (TCP/IP) has been studied in [13]. However, the corresponding studies are mainly focused on stationary networks, i.e., the link qualities do not change dynamically and the optimization problem is for only one snapshot. It is straightforward to extend these techniques to cognitive radio networks operating in relatively stationary spectrum bands, like TV band. However, for spectrum bands with dynamic occupancies of primary users, we face the following two new challenges:

- Dynamic Interruptions: Since a secondary user must quit the corresponding frequency band, each emergence of a

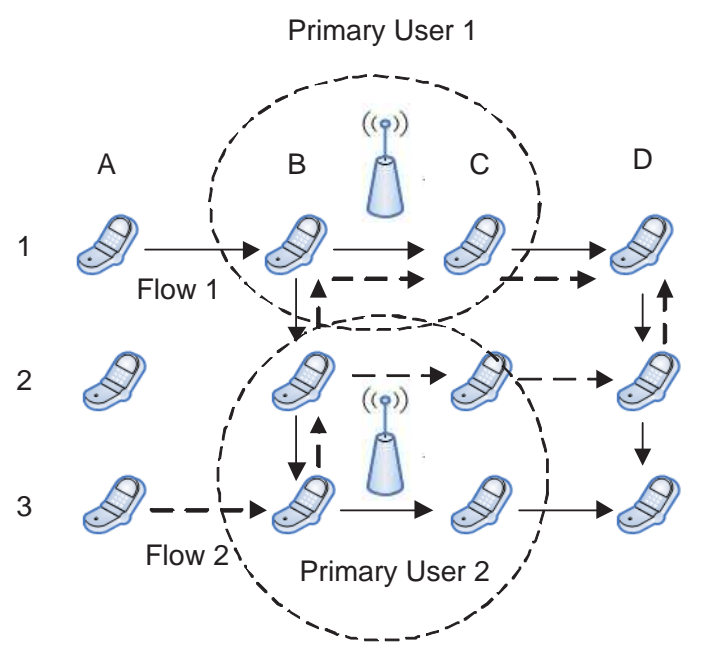

Fig. 1. Illustration of cognitive radio network subject to interruptions.

primary user causes an interruption to the data traffic of secondary users within its interruption range. Even if the secondary users can look for new channels, the procedure of channel switching and sensing new channels also incurs a significant overhead. If the traffic of primary users is bursty, the interruptions are dynamic and random, thus necessitating the stochastic control of the cognitive radio network.

- Medium Time Scale: In many situations, the time scale of interruptions from primary users is medium, which incurs trouble to the strategy optimization. For small time scale (say, a few milliseconds), similar to fast fading, the randomness can be alleviated in the physical layer, e.g. using channel coding to correct errors. Therefore, the negative effect can be smoothed out with time and there is no need for re-routing. For large time scale (say, ten minutes), the optimal route and the transport layer rate can be used for a long period of time. A re-routing or adjustment of transmission rate is infrequent and incurs little overhead. However, for a medium time scale, it is impossible to alleviate the interruptions using approaches like channel coding and it is inefficient to stick to the same transmission rates and routes. 
In this paper, we tackle the problem of joint congestion control and routing in cognitive radio networks subject to the above problems. The procedure of congestion control (i.e., adjusting data rate) and routing (i.e., the decisions of re-routing and path selection) are coupled with each other. Therefore, a joint optimization is needed. Due to the dynamic interruptions and the medium time scale, a stochastic control framework is applied to the joint congestion control and routing. Then, the challenge is how to derive the optimal or near-optimal strategy in various situations. For the centralized case, dynamic programming and $Q$-learning are both applied for the cases with and without primary user information, respectively. For the decentralized case, the stochastic control is decomposed to individual controls via Lagrange pricing. In sharp contrast to one-stage optimizations in stationary networks, the strategies of individual data flows are still coupled even though the capacity constraint has been decoupled via pricing. A heuristic scheme based on a limited lookahead policy and binary pricing scheme is then proposed to break the coupling. Note that the study in this paper does not concern the detailed design of protocols. However, it provides insights and tools for the design of practical cognitive radio networks.

The remainder of this paper is organized as follows. The system model for cognitive radio networks will be introduced in Section II. The elements of control, primal optimization and dual optimization are discussed in Sections III, IV and V, respectively. Numerical results and conclusions are provided in Sections VI and VII, respectively.

\section{System MODEL}

We consider a cognitive radio network, in which there are $L$ cognitive radio links and $N$ data flows. We denote by $x_{s}(t)$ the rate of data flow $s$ at (discrete) time $t$. The rates are stacked into one vector $\mathbf{x}(t)=\left(x_{1}(t), \ldots, x_{N}(t)\right)$. The utility of flow rate $x_{s}$ is given by $U_{s}\left(x_{s}\right)$, where $U_{s}$ is the utility function of data flow $s$. The time is divided into routing periods. We assume that each routing period is sufficiently long such that the rate allocation can be completed within one routing period. The routes of different data flows at routing period $t$ are represented by a matrix $\mathbf{R}(t)$, where the rows stand for links and columns mean data flows. Data flow $j$ passes through link $i$ if the element $R_{i j}(t)$ equals 1 . Otherwise, $R_{i j}(t)=0$. We assume that there are $N_{s}$ possible routes for data flow $s$. We assume that the capacity of link $i$ is given by $c_{i}$. We use one vector, $\mathbf{c}=\left(c_{1}, \ldots, c_{L}\right)$, to denote the capacities of all links. For simplicity, we consider the thermal-limited regime and ignore the coupling of interference. When the interference is strong, we can consider joint congestion control, routing and scheduling. However, it is beyond the scope of this paper.

We use a vector $\mathbf{m}=\left(m_{1}, \ldots, m_{L}\right)^{T}$ to represent primary users' activities. When $m_{i}=1$, link $i$ is not occupied by primary users and can be used by secondary users; otherwise, $m_{i}=0$. For simplicity, we assume that the spectrum occupancy is constant within each routing period. The activity of each primary user is modeled as a two-state Markov chain. The two states are busy (B), i.e., occupied by primary users, and idle (I), i.e., not occupied by primary users. The state transition probabilities are denoted by $P_{s_{1} s_{2}}$, where $s_{1}$ and $s_{2}$ are two consecutive states.

The re-routing procedure may be activated by the emergence of primary user, which may block the corresponding data flow for a long period of time. However, the re-routing may incur unnecessary overhead if the primary user's interruption is actually short. We assume that each re-routing procedure takes $T_{r r}$ routing periods, i.e., only after $T_{r r}$ routing periods can the data flow resume operation. We also assume that each re-routing incurs a penalty $P_{r r}$ since a lot of information exchange is needed for the re-routing procedure.

\section{BASic Elements of the Stochastic Control}

For the joint congestion control and routing in cognitive radio networks subject to dynamic interruptions of primary users, we adopt the framework of optimization based crosslayer design [2] [5] [6]. For simplicity, we consider only single-channel case here. For multi-channel case, we can extend the action space to incorporate channel selection. In this section, we explain the three elements in the joint congestion control and routing, namely reward function, state space and action space.

\section{A. Reward Function}

We consider the discounted sum of all data flows from routing period 1 to routing period $T$. The optimization problem is thus given by

$$
\begin{aligned}
& \max \sum_{t=1}^{T} \beta^{t-1} r_{t} \\
& \text { s.t. } \quad \mathbf{R}(t) \mathbf{x}(t) \leq \mathbf{c} \odot \mathbf{m}(t),
\end{aligned}
$$

where $r_{t}$ is the network-wide reward obtained at routing period $t$, which is given by

$$
r_{t}=\sum_{s=1}^{N} U_{s}\left(x_{s}(t)\right) \phi_{s}(t)-P_{r r} \sum_{s=1} \theta_{s}(t) .
$$

where $0<\beta<1$ is a discounting factor, $\theta_{s}(t)$ is the characteristic function of the event that re-routing begins at $t$, the function $\phi_{s}(t)$ indicates whether the data flow is in active state and $\odot$ means elementwise multiplication, i.e., the capacities of all links are modulated by the spectrum occupancy vector $\mathbf{m}(t)$. Obviously, the first term in the reward function is the reward of data flow and the second term is the penalty for re-routing.

\section{B. State Space}

The system state contains the states of spectrum occupancies of all links and the local states of data flows. For data flow $s$, there are $N_{s}+T_{r r}$ states, namely $N_{s}$ active states, denoted by $\left\{\mathbb{A}_{p}\right\}_{p=1, \ldots, N_{s}}\left(\mathbb{A}_{p}\right.$ means that data flow is active and is using route $p$ ), and $T_{r r}$ re-routing states, denoted by $\left\{\mathbb{R}_{t}\right\}_{t=1, \ldots, T_{r r}}$ ( $t$ means the number of routing periods that have been passed for re-routing). The local state transition of a data flow is illustrated in Fig. 2. 


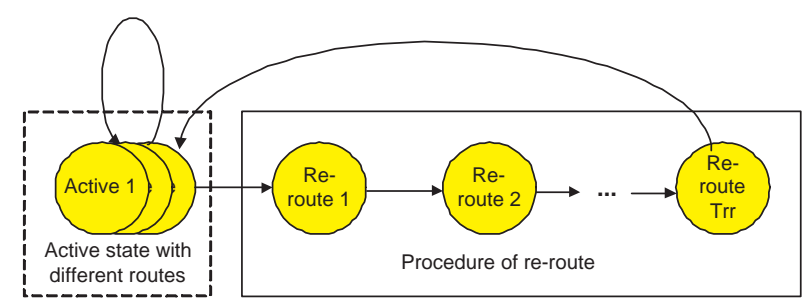

Fig. 2. Illustration of local state transitions.

\section{Action Space}

Each data flow can choose different actions for the following two local states: (a) Active $\mathbb{A}_{p}$ : when the current state is active, each data flow can either adjust its data rate $x_{s}$, or the data flow can choose re-routing, thus entering local state $\mathbb{R}_{1}$; (b)Rerouting $\mathbb{R}_{t}$ : if $t<T_{r r}$, the data flow can do nothing but resuming the re-routing procedure; when $t=T_{r r}$, the data flow should choose the corresponding routing path $p$ and then enter the local active state $\mathbb{A}_{p}$. Note that the states of spectrum occupancies of all links cannot be changed by the actions of data flows since they are affected by only primary users. The actions can change only the local states of data flows.

\section{Centralized Primal Optimization}

In this section, we discuss the centralized primal optimization problem by using dynamic programming and $Q$-learning. We suppose that the optimization is carried out by a control center.

\section{A. Dynamic Programming}

We assume that the knowledge of primary users, i.e., the transition probabilities of the two-state Markov model, is perfectly known. It is well known that the optimal strategy of the stochastic control can be obtained via dynamic programming. Define the value function to be

$$
V_{t}\left(\mathcal{S}_{t}\right)=\max _{\mathbf{R}(t) \mathbf{x}(t) \leq \mathbf{c} \odot \mathbf{m}(t)} \sum_{\tau=t}^{T} \beta^{\tau-1} r_{\tau},
$$

where $\mathcal{S}_{t}$ is the overall system state at routing period $t$. Then, the value functions are given by the Bellman's equation, which is given by

$$
V_{t}\left(\mathcal{S}_{t}\right)=\max _{\mathbf{a}} E\left[\max _{\mathbf{R}(t) \mathbf{x}(t) \leq \mathbf{c} \odot \mathbf{m}(t)} r_{t}+\beta V_{t+1}\left(\mathcal{S}_{t+1}\right)\right],
$$

where the expectation is over the the randomness of the next system state and a means the decisions of re-routing of all data flows. Since the allocation of data flow rate $\mathbf{x}$ does not affect the state transition, it is independent of $V_{t+1}\left(\mathcal{S}_{t+1}\right)$ and is used to optimize the instantaneous reward $r_{t}$. We can use either centralized or decentralized approaches, e.g. pricing based optimization decomposition, to obtain the optimal $\mathrm{x}$ within one routing period. The optimal strategy is then obtained by solving (4), beginning from time $T$.

\section{B. Q-learning}

Note that dynamic programming requires perfect knowledge about primary users. When such a knowledge is unknown, we can apply $Q$-learning. We set $Q$-values for each data flow $s$, system state $\mathcal{S}$ and action $a$, denoted by $Q_{s}(\mathcal{S}, a)$. Then, the learning procedure of different data flows is given by

$$
\begin{aligned}
Q_{s}^{t+1}\left(\mathcal{S}_{t}, a\right) & =(1-\alpha(t)) Q_{s}^{t}\left(\mathcal{S}_{t}, a\right) \\
& +\alpha(t)\left(r_{s}(t)+\max _{u} \beta Q_{s}^{t}\left(\mathcal{S}_{t+1}, u\right)\right),
\end{aligned}
$$

where $\alpha(t)$ is a learning factor. The probability of using action $a$ is given by the following Boltzman distribution:

$$
P_{s}^{t}(\mathcal{S}, a)=\left(e^{\frac{Q_{s}^{t}(\mathcal{S}, a)}{T}}\right) /\left(\sum_{u} e^{\frac{Q_{s}^{t}(\mathcal{S}, u)}{T}}\right) .
$$

\section{Dual Optimization and Decomposition}

In this section, we discuss the dual optimization and propose a heuristic algorithm based on Limited Lookahead Policy (LLP) and binary pricing. We assume that the a priori information about primary users is perfectly known.

\section{A. Dual Optimization}

In order to decompose the stochastic optimization problem, we convert the primal optimization problem into the dual one:

$$
\begin{aligned}
\Lambda^{*}= & \arg \min _{\Lambda} \sum_{s=1}^{N} \max \sum_{t=1}^{T}\left(U_{s}\left(x_{s}(t)\right) \phi_{s}(t)-P_{r r} \theta_{s}(t)\right. \\
& \left.-x_{s}(t) \sum_{l} R_{l s}(t) \lambda_{l}(t)\right)+\sum_{t=1}^{T} \sum_{l} c_{l} m_{l}(t) \lambda_{l}(t),
\end{aligned}
$$

where $\lambda_{l}(t)$ is the Lagrange factor (price) of link $l$ at time $t$ and $\Lambda$ is the set of link prices at different routing periods.

Eq. (7) can be rewritten as

$$
\Lambda^{*}=\arg \min _{\Lambda} G(\Lambda)
$$

where

$$
G(\Lambda)=\sum_{s=1}^{N} V_{s}(\Lambda, T)+\sum_{t=1}^{T} \sum_{l} c_{l} m_{l}(t) \lambda_{l}(t),
$$

and

$$
\begin{aligned}
V_{s}(\Lambda, T) & =\max \sum_{t=1}^{T}\left(U_{s}\left(x_{s}(t)\right) \phi_{s}(t)\right. \\
& \left.-P_{r r} \theta_{s}(t)-x_{s}(t) \sum_{l} R_{l s}(t) \lambda_{l}(t)\right) .
\end{aligned}
$$

Obviously, $V_{s}\left(\Lambda_{s}, T\right)$ means the optimal strategy of data flow $s$, given link prices $\Lambda$. In stationary systems, $V_{s}(\Lambda, T)$ can be optimized by data flow $s$ without coupling with other data flows. However, in the dynamic environment due to interruptions from primary users, the decomposition is no longer valid since the action taken by a data flow depends on the current system state, which couples with the actions of other data flows. Therefore, the dual optimization based decomposition in traditional cross-layer design does not apply 
in the stochastic control of cognitive radio networks. Although there exist some approaches to decompose stochastic control into subproblems, e.g. scenario tree based stochastic programming [8] and Uzawa-based heuristic algorithm [1], the former is mainly used to decompose large scale linear programming problems instead of tackling utility privacies, while the latter needs a predetermined function to describe the change of price without a general expression. A systematic approach to decompose the stochastic control with utility privacy is still an open problem. Therefore, we consider only a heuristic suboptimal approach in the next subsection.

\section{B. LLP Strategy and Binary Pricing}

When each secondary user knows only its local state, it is difficult for secondary users to optimize its strategy since the expected payoff is dependent on the system state, as well as other secondary users' strategies. In this case, we let secondary users adopt a LLP strategy, i.e. looking ahead for only limited steps.

When all links of a data flow are not interrupted by primary users, there is no need to carry out re-routing. When one (or more) link in the data flow is interrupted by primary users, the data flow needs to consider whether carry out a re-routing procedure. Due to the LLP strategy, the data flow compares the actions of keeping current route and changing the route, respectively. If a re-routing procedure is initiated, the loss is given by

$$
L_{1}=P_{r r}+T_{r r} \bar{U}
$$

where $\bar{U}$ is the expected utility of traffic in each routing period. We use the utility of traffic averaged over all previous routing rounds to approximate $\bar{U}$. If no re-routing procedure is carried out and the data flow waits for the recovery of the whole path, the expected loss is given by

$$
L_{2}=\bar{T}\left(\sum_{i \in R_{i}} \lambda_{i}+\bar{U}\right),
$$

where $\bar{T}$ is the expected time needed for primary users to quit the spectrum, which can be obtained from $P_{B I}$.

It is quite challenging to find the optimal pricing since it is coupled with all secondary data flows. Therefore, we use a simple binary pricing strategy, i.e. for link $i$, the price $\lambda_{i}$ is 0 when the link is available; otherwise, it is $\lambda_{h}$, which is common for all links. Numerical simulation will show that such a simple pricing strategy achieves near-optimal performance.

\section{Numerical Results}

We consider the network illustrated in Fig. 1, in which the nodes are labeled using their coordinates, e.g. A1 or B2. We assume that there are two data flows, namely $\mathrm{A} 1 \rightarrow \mathrm{D} 3$ and A $3 \rightarrow$ D1. Each data flow has two possible routes, each having 5 hops:

- Path 1 for data flow 1: $\mathrm{A} 1 \rightarrow \mathrm{B} 1 \rightarrow \mathrm{C} 1 \rightarrow \mathrm{D} 1 \rightarrow \mathrm{D} 2 \rightarrow \mathrm{D} 3$.

- Path 2 for data flow $1: \mathrm{A} 1 \rightarrow \mathrm{B} 1 \rightarrow \mathrm{B} 2 \rightarrow \mathrm{B} 3 \rightarrow \mathrm{C} 3 \rightarrow \mathrm{D} 3$.
- Path 1 for data flow 2: $\mathrm{A} 3 \rightarrow \mathrm{B} 3 \rightarrow \mathrm{B} 2 \rightarrow \mathrm{B} 1 \rightarrow \mathrm{C} 1 \rightarrow \mathrm{D} 1$.

- Path 2 for data flow 2: $\mathrm{A} 3 \rightarrow \mathrm{B} 3 \rightarrow \mathrm{B} 2 \rightarrow \mathrm{C} 2 \rightarrow \mathrm{D} 2 \rightarrow \mathrm{D} 1$.

We assume that a re-routing procedure has penalty $P_{r r}=1$ and duration $T_{r r}=5$. Therefore, each data flow has 7 local states. The channel capacity of each link is set to 1 except for the link between D1 and D2, which is set to 2. Obviously, the optimal scheme is to let data flow 1 choose path 1 and data flow 2 choose path 2 , which yields throughput 1 for both data flows. We assume $T \rightarrow \infty$, i.e. we consider an infinite time horizon.

Suppose that there are two primary users co-existing with the cognitive radio network. Their locations and the ranges of interruptions are illustrated in Fig. 1. We consider the following two cases of the primary users' activity: (a) Case 1: for primary user $1, P_{I B}=0.01$ and $P_{B I}=0.05$; for primary user $2, P_{I B}=0.02$ and $P_{B I}=0.1$; (b) Case 2: for primary user $1, P_{I B}=0.1$ and $P_{B I}=0.25$; for primary user 2, $P_{I B}=0.15$ and $P_{B I}=0.3$.

Obviously, in Case 1, the channel occupancy is more stationary and each interruption from primary users is much longer than that of Case 2. Since the primary users have 4 states and each data flow has 7 states, there are totally 196 system states.

We consider the data throughput as the utility $U$. Therefore, the objective is to maximize the discounted total throughput, subtracting the penalty of re-routing. When the routes are fixed and the spectrum occupancy is known, the optimal rate assignment $\left\{x_{s}\right\}$ can be efficiently obtained via linear programming.

\section{A. Optimal Strategies via Dynamic Programming}

We used dynamic programming to compute the optimal strategies for cases 1 and 2. The optimal actions of some key system states are listed in Table I for case 1. The results for case 2 are omitted due to the limited space and the similar conclusions. The notation of actions is explained as follows: A (continue re-routing), B (begin re-routing), C (keep current route), D (choose route 1), E (choose route 2) and F (stay in the active state).

From both results, we find the following strategy differences which coincide with intuitions: (a) In Case 1, whenever one or more primary user emerges, the corresponding data flow begins re-routing, since the duration of primary user occupancy is long. (b) In Case 2, the data flows do not respond to the primary users' emergence. Re-routing is carried out when the data flows are not using the optimal path, e.g., when data flow 1 is using Path 2 and data flow 2 is using Path 1.

\section{B. Q-Learning}

For both cases 1 and 2, we applied $Q$-learning to learn the optimal strategy without the knowledge of $P_{I B}$ and $P_{B I}$. The results are provided in Fig. 3, in which each epoch means 200 routing periods and the rewards are computed using the 200 instantaneous rewards in the corresponding epoch. We observe that, in both cases, the $Q$-learning can effectively improve the performance. The learning speed of Case 2 is faster since the 
TABLE I

The Optimal StRategy for Typical System States IN CASE 1

\begin{tabular}{ccccc}
\hline & $(0,0)$ & $(0,1)$ & $(1,0)$ & $(1,1)$ \\
\hline$(1,1)$ & $(\mathrm{B}, \mathrm{B})$ & $(\mathrm{B}, \mathrm{B})$ & $(\mathrm{C}, \mathrm{B})$ & $(\mathrm{C}, \mathrm{B})$ \\
\hline$(1,2)$ & $(\mathrm{B}, \mathrm{B})$ & $(\mathrm{B}, \mathrm{C})$ & $(\mathrm{C}, \mathrm{B})$ & $(\mathrm{C}, \mathrm{C})$ \\
\hline$(1,3)$ & $(\mathrm{B}, \mathrm{A})$ & $(\mathrm{B}, \mathrm{A})$ & $(\mathrm{C}, \mathrm{A})$ & $(\mathrm{C}, \mathrm{A})$ \\
\hline$(1,7)$ & $(\mathrm{B}, \mathrm{E})$ & $(\mathrm{B}, \mathrm{E})$ & $(\mathrm{C}, \mathrm{E})$ & $(\mathrm{C}, \mathrm{E})$ \\
\hline$(2,1)$ & $(\mathrm{B}, \mathrm{B})$ & $(\mathrm{C}, \mathrm{B})$ & $(\mathrm{B}, \mathrm{B})$ & $(\mathrm{B}, \mathrm{C})$ \\
\hline$(2,2)$ & $(\mathrm{B}, \mathrm{B})$ & $(\mathrm{B}, \mathrm{C})$ & $(\mathrm{B}, \mathrm{B})$ & $(\mathrm{B}, \mathrm{C})$ \\
\hline$(2,3)$ & $(\mathrm{B}, \mathrm{A})$ & $(\mathrm{C}, \mathrm{A})$ & $(\mathrm{B}, \mathrm{A})$ & $(\mathrm{C}, \mathrm{A})$ \\
\hline$(2,7)$ & $(\mathrm{B}, \mathrm{E})$ & $(\mathrm{C}, \mathrm{E})$ & $(\mathrm{B}, \mathrm{E})$ & $(\mathrm{C}, \mathrm{E})$ \\
\hline$(3,1)$ & $(\mathrm{A}, \mathrm{B})$ & $(\mathrm{A}, \mathrm{B})$ & $(\mathrm{A}, \mathrm{B})$ & $(\mathrm{A}, \mathrm{C})$ \\
\hline$(3,2)$ & $(\mathrm{A}, \mathrm{B})$ & $(\mathrm{A}, \mathrm{C})$ & $(\mathrm{A}, \mathrm{B})$ & $(\mathrm{A}, \mathrm{C})$ \\
\hline$(3,3)$ & $(\mathrm{A}, \mathrm{A})$ & $(\mathrm{A}, \mathrm{A})$ & $(\mathrm{A}, \mathrm{A})$ & $(\mathrm{A}, \mathrm{A})$ \\
\hline$(7,1)$ & $(\mathrm{D}, \mathrm{B})$ & $(\mathrm{D}, \mathrm{B})$ & $(\mathrm{D}, \mathrm{B})$ & $(\mathrm{D}, \mathrm{C})$ \\
\hline$(7,2)$ & $(\mathrm{D}, \mathrm{B})$ & $(\mathrm{D}, \mathrm{C})$ & $(\mathrm{D}, \mathrm{B})$ & $(\mathrm{D}, \mathrm{C})$ \\
\hline$(7,7)$ & $(\mathrm{D}, \mathrm{F})$ & $(\mathrm{D}, \mathrm{F})$ & $(\mathrm{D}, \mathrm{F})$ & $(\mathrm{D}, \mathrm{F})$ \\
\hline & & & &
\end{tabular}

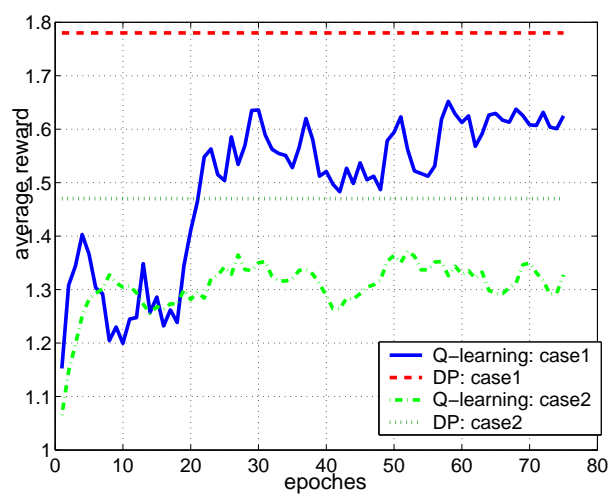

Fig. 3. Comparison of performances of dynamic programming and $Q$ learning.

primary users emerge more frequently, thus providing more experience for learning. Anyhow, both learning procedures are slow, which implies the importance of the a priori knowledge of primary users.

\section{LLP Strategy and Binary Pricing}

For both cases 1 and 2, we test the performance for both cases 1 and 2 using different prices, ranging from 0.1 to 1.5. The results are provided in Fig. 4. We observe that, for Case 1, the LLP strategy achieves almost perfect performance, regardless of the price; for Case 2, when the price is properly chosen, the performance of the LLP is also close to that achieved by dynamic programming. This demonstrates that the proposed LLP approach is near-optimal.

\section{CONCLUSIONS}

We have studied the joint congestion control and routing in cognitive radio networks suffering from the dynamic interruptions of primary users. Due to the time variation of spectrum occupancy, stochastic control is applied for the strategy of congestion control and routing. Multiple approaches like dynamic programming, $Q$-learning and pricing based algorithm, have been applied to obtain the stochastic control strategies in various scenarios. Their performances have been demonstrated by numerical simulations.

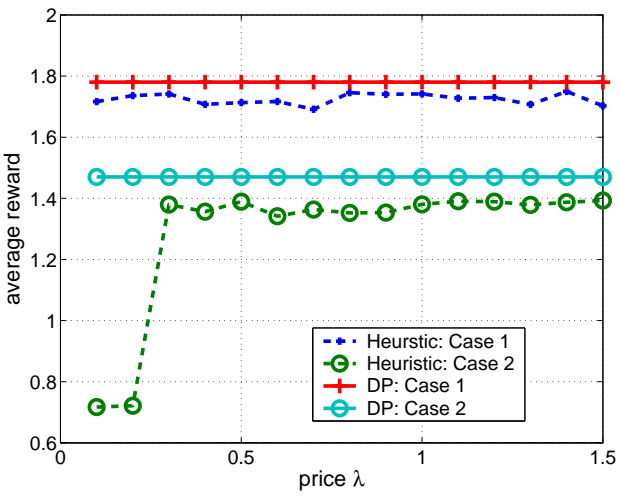

Fig. 4. Comparison of performances of dynamic programming and LLP strategy/binary pricing.

\section{ACKNOWLEDGMENT}

This research work is supported in part by NSF under CNS1040207, ECCS-0901425, and the US Army Research Office.

\section{REFERENCES}

[1] K. Barty, P. Carpentier and P. Girardeau, "Decomposition of large-scale stochastic optimal control problems," preprint, 2009.

[2] M. Chiang, S. H. Low, A. R. Calderbank and J. C. Doyle, "Layering as optimization decomposition: A mathematical theory of network architectures," Proceedings of the IEEE, vol.95, pp.255-312, 2007.

[3] K.R. Chowdhury, M.D. Felice and I.F. Akyildiz, "TP-CRAHN: A transport protocol for cognitive radio ad-hoc networks," in Proc. of IEEE Conference on Computer Communications (Infocom), April. 2009.

[4] J. Jia, Q. Zhang and X. Shen, "HC-MAC: A hardware-constrained cognitive MAC for efficient spectrum management," IEEE Journal on Selected Areas in Communications, vol. 26, pp. 106-117, Jan. 2008.

[5] F. Kelly, "Charging and rate control for elastic traffic," European Transactions on Telecommunications, vol.8, pp.33-37, 1997.

[6] F. Kelly, A. Maullo and D. Tan, "Rate control in communication networks: Shadow prices, proportional fairness and stability," Journal of the Operational Research Society, vol.49, pp.237-252, 1998.

[7] H. Ma, L. Zheng and X. Ma, "Spectrum-aware routing for multihop cognitive radio networks with a single transceiver," in Proc. of the Cognitive Radio Oriented Wireless Networks and Communications (CrownCom), May. 2008.

[8] A. Ruszczyński and Shapiro (edt.), Stochastic Programming, Handbooks in Operations Research and Management Science, vol.10, Elsevier, 2003.

[9] A. Sampath, L. Yang, L. Cao, H. Zheng and B.Y. Zhao, "High throughput spectrum-aware routing for cognitive radio based ad hoc networks," in Proc. of the International Conference on Cognitive Radio Oriented Wireless Networks and Communications (CrownCom), May. 2008.

[10] Y. Shi and Y. T. Hou, "A distributed optimization algorithm for multiHop cognitive radio networks," in Proc. of IEEE Conference on Computer Communications (Infocom), April. 2008.

[11] H. Su and X. Zhang, "Cross-Layer based opportunistic MAC protocols for QoS provisionings over cognitive radio wireless networks," IEEE Journal on Selected Areas in Communication, vol. 26, pp. 118-129, Jan. 2008.

[12] R. Urgaonkar and M. J. Neely, "Opportunistic scheduling with reliability guarantees in cognitive radio networks," IEEE Trans. Mobile Computing, vol. 8, pp. 766-777, June. 2009.

[13] J. Wang, L. Li, S. H. Low and J. C. Doyle, "Cross-layer optimization in TCP/IP networks," IEEE/ACM Trans. Netw., vol.13, no.3, pp.582-586, June 2005.

[14] Q. Wang and H. Zheng, "Route and spectrum selection in dynamic spectrum networks," in Proc. of IEEE Consumer Communications Communications and Networking Conference (CNCC), Jan. 2006. 